\title{
Multi-Sensor Multi-Target Tracking Using Domain Knowledge and Clustering
}

\author{
Shaoming He, Hyo-Sang Shin*, Antonios Tsourdos
}

\begin{abstract}
This paper proposes a novel joint multi-target tracking and track maintenance algorithm over a sensor network. Each sensor runs a local joint probabilistic data association (JPDA) filter using only its own measurements. Unlike the original JPDA approach, the proposed local filter utilises the detection amplitude as domain knowledge to improve the estimation accuracy. In the fusion stage, the DBSCAN clustering in conjunction with statistical test is proposed to group all local tracks into several clusters. Each generated cluster represents the local tracks that are from the same target source and the global estimation of each cluster is obtained by the generalized covariance intersection (GCI) algorithm. Extensive simulation results clearly confirms the effectiveness of the proposed multisensor multi-target tracking algorithm.
\end{abstract}

Index Terms-Multi-sensor multi-target tracking, Joint probabilistic data association, Detection amplitude, DBSCAN clustering

\section{INTRODUCTION}

Proliferation of low-cost sensors and recent advances in wireless sensor technology inspired the employment of multiple sensor nodes, capable of communicating with each other. With respect to the single sensor target tracking, utilisation of a sensor network can significantly improve the sensor coverage and the estimation accuracy, enabling complicated sensing and tracking tasks [1], [2]. Unfortunately, the quality of low-cost sensors is relatively poor compared with the high performance sensors. Thus, such a sensor is subject to a certain degree of uncertainty, resulting in relatively high miss-detections and false alarm rates. This could become exacerbated in multitarget tracking (MTT) scenarios, where the mappings between the targets and the measurements are unknown [3], [4]. The number of targets in the surveillance area of interest is also not known a priori in a typical MTT problem.

This paper aims to develop a multi-target tracking algorithm using a network of low-cost sensors that are subject to a certain degree of uncertainties. In general, the multi-sensor multitarget tracking consists of two stages: the first stage is a local multi-target tracking phase and the second is the estimation fusion phase. The focus of this paper is to develop efficient algorithms for handling important issues in both stages.

In the local estimation stage, each sensor node runs a MTT algorithm to obtain the local tracks. As discussed, the key issue is that the measurement uncertainty could significantly degrade the performance of MTT. Data association is a plausible and widely-accepted solution in MTT to resolve

Shaoming He, Hyo-Sang Shin and Antonios Tsourdos are with the School of Aerospace, Transport and Manufacturing, Cranfield University, Cranfield MK43 0AL, UK (email: \{shaoming.he,h.shin,a.tsourdos\}@ cranfield.ac.uk)

*Corresponding author the problem of measurement uncertainty. This technique discerns target-generated measurements from clutters and finds the mappings between targets and measurements. Multiple hypothesis tracking (MHT) is one of the most well-known association algorithms and a well-established paradigm [5][7]. Fundamentally, MHT maintains all incompatible (e.g., unresolved) track hypotheses in track trees during a sliding window. A decision is made to remove the impossible hypotheses until enough information is available, provided by the observations. At each time instant, MHT utilises the Maximum a Posterior scheme (e.g., finding the most probable global hypothesis) for track update. Although MHT is widely accepted as the most powerful MTT solution, the inherent multi-scan data association problem is NP-hard due to the explosion of hypotheses. Therefore, the optimal solution of MHT is computationally intractable and approximations are required for practical implementation [8]-[10].

Another alternative of data association is JPDA filter, which is known as a sub-optimal minimum-mean-squarederror (MMSE) estimator for MTT. Unlike MHT, JPDA allows for the possibility that a measurement can be associated to a number of candidate targets within the validation gate. Since all of the potential candidates are considered in data association, JPDA is particularly advantageous in MTT, especially for dense clutter scenarios, and shows wide range of applications in sonar-based tracking [11], computer vision [12], space object tracking [13], [14], cell tracking [15], radarbased tracking [16], etc. Compared to MHT, JPDA can achieve reasonable tracking accuracy with lower computational burden [11].

Considering the balance between the sensitivity issue and the computational cost, this paper adopts the JPDA concept as the underlying data association approach and proposes a modified JPDA algorithm. Unlike the typical JPDA algorithms, the proposed JPDA algorithm incorporates additional domain knowledge in obtaining the measurement likelihood for data association and track initialisation. Note that most MTT algorithms utilise only the kinematics measurement, i.e., position, angle and range, for track update. It is certain that additional domain knowledge can be utilised to improve the tracking performance. In radar applications, for example, the target detection is achieved by thresholding the signal amplitude [17], [18]. This amplitude feature, therefore, can be taken as the domain knowledge and exploited in the development of tracking algorithms to enhance tracking quality and continuity. The rational behind this is that the received measurement with its amplitude higher than a pre-specified threshold is more likely to be a target-generated measurement. The signal 
amplitude information is also helpful in track initialisation and data association in a low signal-to-noise ratio (SNR) environment [19], which is common for low-cost sensors. Therefore, the additional domain knowledge incorporated in this paper is the signal amplitude information. Leveraging this information can drastically reduce the number of false tracks for low SNR targets, consequently improving the target tracking performance.

In the fusion stage, the sensor nodes communicate with each other to perform estimation fusion through a sensor network topology. Although the sensor fusion technology for single target tracking is well-established, a direct extension of these fusion algorithms to a multi-target tracking scenario is not straightforward. In multi-target tracking cases, trackto-track association is essential to find the target source of local tracks that come from different sensors. Track-to-track association, similar to measurement-to-track association in MHT, is a multidimensional assignment problem, known as $S-D$ ( $S$-dimensional) assignment, where $S$ refers to the number of sensors [20]-[25].

The main challenge in associating data from three or more sensors is that the resulting multidimensional assignment problem is NP-hard. Due to this intrinsic property, finding optimal solution of the $S-D$ assignment problem is intractable even for fairly small-sized problems. The mainstream suboptimal solution of the $S-D$ assignment in track-to-track association is the well-established Lagrangian relaxation approach [26] [28]. However, this algorithm too can become inefficient for fairly large scenarios, since it involves iteratively solving a $2-D$ assignment problem to produce better solutions to the original $S-D$ assignment problem. Therefore, it is imperative and meaningful to find an alternative solution of the $S-D$ assignment problem.

To this end, this paper also proposes a new tractable trackto-track association and fusion algorithm for multi-sensor multi-target tracking. To find the target origin of the local tracks at the fusion stage, we utilise the clustering concepts and develop a modified DBSCAN clustering algorithm. The proposed clustering algorithm alleviates the issue of computational tractability in track-to-track association while retaining reasonable tracking performance. The analysis shows that the proposed track-to-track algorithm is less computationally complex compared with the exact $S-D$ algorithm and the well-known Lagrangian relaxation approach. After clustering, the fusion centre obtains the global fused estimation of each cluster by using the GCI technique.

The contributions of this paper are twofold. On one hand, a new domain knowledge-aided JPDA filter is proposed for MTT to improve the estimation performance in a low SNR environment. On the other hand, the track-to-track association problem is solved through a modified DBSCAN clustering algorithm in a computationally efficient way. The performance characteristics of the proposed multi-sensor multi-target tracking scheme are investigated through extensive empirical tests. We define simulation scenarios with a low SNR to validate the performance of the proposed approach in the case where local sensors in a network are subject to a certain level of uncertainties. For rigorous evaluation, the performance of the proposed approach is also compared with other well-known approaches.

The rest of the paper is organised as follows. Sec. II presents some preliminaries this study. Sec. III provides the details the proposed feature enhanced local JPDA filter, followed by the track-to-track fusion shown in Sec. IV. Finally, some simulations and conclusions are offered. Throughout the paper, we use the symbol $i$ to refer to the target index, $j$ the measurement index, and $l$ the sensor index.

\section{PRoBlem Formulation}

\section{A. Multi-Target Model}

Suppose at scan $k$ of there are $N_{k}$ targets and $M_{k}$ sensor measurements, A multi-target state and a multi-target measurement are then defined by random finite sets as

$$
X_{k}=\left\{x_{k}^{1}, \ldots, x_{k}^{N_{k}}\right\}, \quad Z_{k}=\left\{z_{k}^{0}, z_{k}^{1}, \ldots, z_{k}^{M_{k}}\right\}
$$

where $x_{k}^{i}$ denotes the $i$ th target at scan $k, z_{k}^{j}(j \neq 0)$ the $j$ th measurement received at scan $k$, and $z_{k}^{0}$ the dummy measurement for convenient representation of miss detection.

We assume that the temporal evolution of each target is independent of each other and follows a Markov transition model $p\left(x_{k}^{i} \mid x_{k-1}^{i}\right)$. Each sensor measurement $z_{k}^{j}$ consists of a kinematic part $\tilde{z}_{k}^{j}$, i.e. position, angle and range, and a detection amplitude part $a_{k}^{j}$, that is, the single measurement vector has the form $z_{k}^{j}=\left[\tilde{z}_{k}^{j T}, a_{k}^{j}\right]^{T}$. To facilitate the design process, we assume that the kinematic measurement is independent of the signal amplitude and thereby the measurement likelihood for the target-generated measurement can be formulated as

$$
p\left(z_{k}^{j} \mid x_{k}^{i}\right)=p\left(z_{k}^{j} \mid x_{k}^{i}\right) p_{1}\left(a_{k}^{j}\right)
$$

where $p_{1}\left(a_{k}^{j}\right)$ denotes the probability density function of the amplitude that is originated from a target.

Similarly, the clutter likelihood $c\left(z_{k}^{j}\right)$ is given by

$$
c\left(z_{k}^{j}\right)=c\left(\tilde{z}_{k}^{j}\right) p_{0}\left(a_{k}^{j}\right)
$$

where $p_{0}\left(a_{k}^{j}\right)$ denotes the probability density function of the amplitude that is due to noise only.

Typically, the amplitude probability density function is parametrised by the SNR and the target SNR is fluctuating randomly subject to four different models, i.e. Swerling type 1, 2, 3, and 4 [29]. In this paper, we adopt the well-known Rayleigh target amplitude model [30] and the amplitude is assumed to be provided by a specific single ping detector. Assuming a normalized background noise, the probability density functions of the amplitude are given by

$$
\begin{gathered}
p_{1}\left(a_{k}^{j}\right)=a_{k} \exp \left(-\frac{\left(a_{k}^{j}\right)^{2}}{2}\right), \quad a_{k}^{j} \geq 0 \\
p_{0}\left(a_{k}^{j}\right)=\frac{a_{k}^{j}}{1+d} \exp \left(-\frac{\left(a_{k}^{j}\right)^{2}}{2(1+d)}\right), \quad a_{k}^{j} \geq 0
\end{gathered}
$$




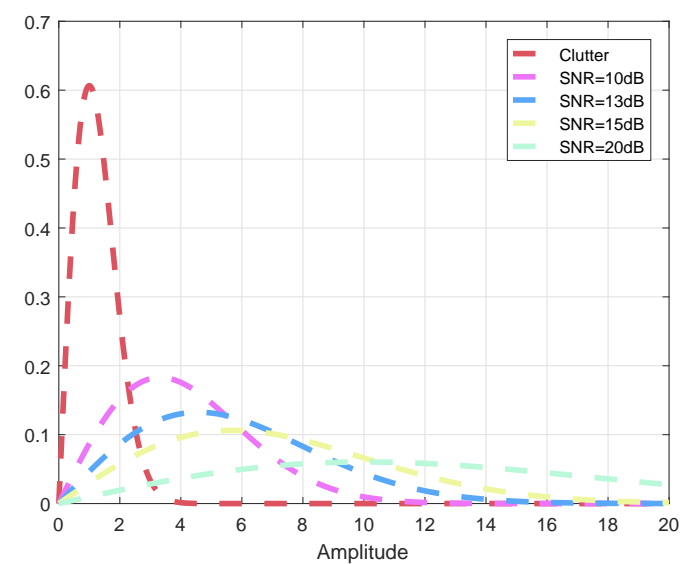

Fig. 1: Amplitude probability density functions of Rayleigh likelihoods for clutter and different SNRs.

where $1+d$ represents the expected (or mean) SNR, which is typically defined in the $\log$ scale $\operatorname{SNR}(d B)=10 \log (1+d)$.

Figure 1 depicts the amplitude probability density functions of Rayleigh likelihoods for clutter and different SNR targets. Each target-generated measurement is independent of others and is detected once the amplitude is larger than a predesignated threshold $\tau$. When thresholding the received signal by $\tau$, the probability of detection $P_{D}$ and the probability of false alarm $P_{F A}$ can, respectively, be obtained as

$$
\begin{gathered}
P_{D}=\int_{\tau}^{\infty} p_{1}\left(a_{k}^{j}\right) d a_{k}^{j} \\
P_{F A}=\int_{\tau}^{\infty} p_{0}\left(a_{k}^{j}\right) d a_{k}^{j}
\end{gathered}
$$

For simplicity, we assume that clutters or false alarms are modelled by a Poisson point process (PPP) with spatial intensity $\lambda_{F A}$ before signal thresholding. Then, $\lambda_{F A} V_{s}$ with $V_{s}$ being the sensor volume provides the expected number of false alarms.

\section{B. Target Existence Model}

In this paper, we assume that target birth is modeled as a PPP with intensity $\lambda_{B}$. Note that by incorporating the PPP birth model into JPDA filter, we can easily utilise the amplitude feature in track initialization, which will dramatically increase the track confirmation speed for low SNR cases. This will be confirmed in the simulations. Each track is confirmed or terminated based on target existence probability $p\left(\chi_{k}^{i} \mid Z_{k-1}\right)$. The time evolution of $\chi_{k}^{i}$ can be formulated by the Markov Chain One model [31].

\section{Algorithm Overview}

The problem considered in this paper involves a set of $N_{s}$ sensors that provide local track estimations of an unknown number of targets using their own observations. These local track estimations are then transmitted to a fusion centre via a communication network to perform track-to-track fusion.

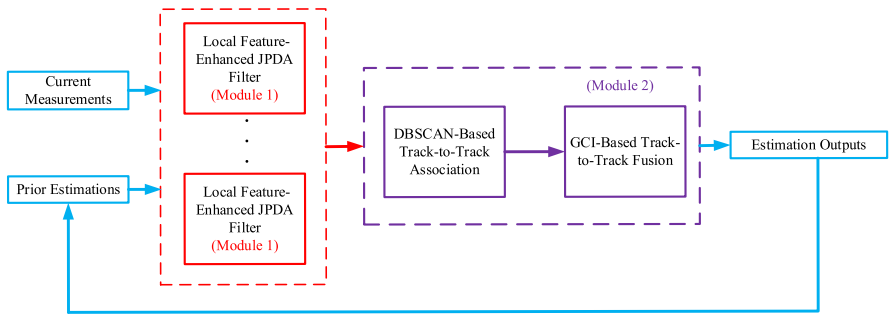

Fig. 2: Information flow of the proposed algorithm.

The proposed multi-sensor multi-target tracking algorithm consists of two modules: (1) feature enhanced local JPDA filter; and (2) track-to-track fusion. In local estimation, each sensor of the sensor network runs an amplitude feature enhanced JPDA filter using its own measurements for the prediction and update stages. Furthermore, a PPP target existence model is also embedded into the local JPDA filter for automatic track maintenance. In the fusion stage, sensors transmit their local estimates to a fusion centre to perform track-to-track association based on the modified DBSCAN clustering method to generate several clusters with each cluster representing the local estimations that originate from the same target. Once the track-to-track association is finished, the global estimation fusion is achieved through the GCI method for each cluster. The information flow of the proposed algorithm is presented Fig. 2.

\section{Feature Enhanced Local JPDA Filter}

This section proposes a feature enhanced local JPDA filter by utilising the amplitude information. For brevity, we ignore the sensor index in this section.

\section{A. Joint Probabilistic Data Association}

The relationship between targets and measurements is assumed to satisfy: (1) each measurement (except for the dummy one) is assigned to at most one target; and (2) each target is uniquely assigned to a measurement. Let $\Theta_{k}=\left\{\theta_{k}^{i}\right\}$, $i \in\left\{1,2, \ldots, N_{k \mid k-1}+M_{k \mid k-1}\right\}$, denote the joint association event. For each pre-existed target $i \in\left\{1,2, \ldots, N_{k \mid k-1}\right\}$, define $\theta_{k}^{i} \in\left\{0,1, \ldots, M_{k}\right\}$ as the association event, and let $\theta_{k}^{i}=j$ denote the $j$ th measurement is originated from the $i$ th target. We create a new track for each measurement $j \in\left\{1,2, \ldots, M_{k \mid k-1}\right\}$ at scan $k$, and the association event for these new targets are defined by $\theta_{k}^{N_{k \mid k-1}+j} \in$ $\left\{1, \ldots, N_{k \mid k-1}+M_{k}\right\}$. That is, if target $N_{k \mid k-1}+j$ is associated with the $j$ th measurement, then $\theta_{k}^{N_{k \mid k-1}+j}=N_{k \mid k-1}+j$. Under the assumption that that each single association event is independent, the MMSE estimate of each target is given by as

$$
p\left(x_{k}^{i} \mid \chi_{k}^{i}, Z_{k}\right)=\sum_{\theta_{k}^{i}} p\left(x_{k}^{i} \mid \theta_{k}^{i}, \chi_{k}^{i}, Z_{k}\right) p\left(\theta_{k}^{i} \mid \chi_{k}^{i}, Z_{k}\right)
$$

In practice, propagation of mixture is computationally intractable and therefore JPDA approximates (8) by a single probability density function based on simple momentpreserving approach. Note that the hypothesis-conditioned 
distribution $p\left(x_{k}^{i} \mid \theta_{k}^{i}, \chi_{k}^{i}, Z_{k}\right)$ can be calculated by standard Kalman filter algorithm. According to Bayesian theory, the marginal association probability $p\left(\theta_{k}^{i} \mid \chi_{k}^{i}, Z_{k}\right)$ is determined by

$p\left(\theta_{k}^{i} \mid \chi_{k}^{i}, Z_{k}\right)=\frac{p\left(\theta_{k}^{i}, \chi_{k}^{i} \mid Z_{k}\right)}{p\left(\chi_{k}^{i} \mid Z_{k}\right)}=\frac{p\left(\chi_{k}^{i} \mid \theta_{k}^{i}, Z_{k}\right) p\left(\theta_{k}^{i} \mid Z_{k}\right)}{p\left(\chi_{k}^{i} \mid Z_{k}\right)}$

where the posterior existence probability is given by

$$
p\left(\chi_{k}^{i} \mid Z_{k}\right)=\sum_{\theta_{k}^{i}} p\left(\theta_{k}^{i}, \chi_{k}^{i} \mid Z_{k}\right)
$$

The joint probability $p\left(\theta_{k}^{i}, \chi_{k}^{i} \mid Z_{k}\right)$ can be obtained as

$$
p\left(\theta_{k}^{i}, \chi_{k}^{i} \mid Z_{k}\right)=p\left(\chi_{k}^{i} \mid \theta_{k}^{i}, Z_{k}\right) p\left(\theta_{k} \mid Z_{k}\right)
$$

According to the law of total probability, $p\left(\theta_{k}^{i} \mid Z_{k}\right)$ can be theoretically calculated by enumerating all possible joint events as $p\left(\theta_{k}^{i}=j \mid Z_{k}\right)=\sum_{\theta_{k}^{i}\left(\in \Theta_{k}\right)=j} p\left(\Theta_{k} \mid Z_{k}\right)$, where the posterior distribution of the joint association event $p\left(\Theta_{k} \mid Z_{k}\right)$ is given by

$$
\begin{aligned}
& p\left(\Theta_{k} \mid Z_{k}\right) \propto\left[\prod_{i \in\left[N_{k \mid k-1}\right], \theta_{k}^{i}=0} 1-P_{D} p\left(\chi_{k}^{i} \mid Z_{k-1}\right)\right] \\
& \times\left[\prod_{i \in\left[N_{k \mid k-1}\right], \theta_{k}^{i}=j} P_{D} p\left(\chi_{k}^{i} \mid Z_{k-1}\right) p\left(z_{k}^{j} \mid x_{k \mid k-1}^{i}\right)\right] \\
& \times\left[\prod_{\theta_{k}^{N_{k \mid k-1}+j}=N_{k \mid k-1}+j} \tilde{\lambda}_{F A}+P_{D} \lambda_{B} p\left(z_{k}^{j} \mid x_{b}\right)\right]
\end{aligned}
$$

where $x_{b}$ represents the possible states of new targets and $\tilde{\lambda}_{F A}$ denotes the pseudo clutter intensity after thresholding. Since a measurement either belongs to an existing confirmed track or an external source (new target or false alarm), Eq, (12) can be reformulated as

$$
\begin{array}{r}
p\left(\Theta_{k} \mid Z_{k}\right) \propto\left[\prod_{i \in\left[N_{k \mid k-1}\right], \theta_{k}^{i}=0} 1-P_{D} p\left(\chi_{k}^{i} \mid Z_{k}\right)\right] \\
\quad \times\left[\prod_{i \in\left[N_{k \mid k-1}\right], \theta_{k}^{i}=j} \frac{P_{D} p\left(\chi_{k}^{i} \mid Z_{k}\right) p\left(z_{k}^{j} \mid x_{k}^{i}\right)}{\bar{\lambda}_{F A}+P_{D} \lambda_{B} p\left(z_{k}^{j} \mid x_{k}^{i}\right)}\right]
\end{array}
$$

Notice that the clutter spatial distribution is given by $c\left(\tilde{z}_{k}^{j}\right)=1 / V_{s}$. Using Eq. (3) gives the intensity of the pseudo clutter as

$$
\tilde{\lambda}_{F A}=\lambda_{F A} V_{s} c\left(z_{k}^{j}\right)=\lambda_{F A} p_{0}\left(a_{k}^{j}\right)
$$

Substituting Eqs. (2) and (14) into (13) yields

$$
\begin{aligned}
p\left(\Theta_{k} \mid Z_{k}\right) \propto\left[\prod_{i \in\left[N_{k \mid k-1}\right], \theta_{k}^{i}=0} 1-P_{D} p\left(\chi_{k}^{i} \mid Z_{k}\right)\right] \\
\quad \times\left[\prod_{i \in\left[N_{k \mid k-1}\right], \theta_{k}^{i}=j} \frac{P_{D} p\left(\chi_{k}^{i} \mid Z_{k}\right) p\left(\tilde{z}_{k}^{j} \mid x_{k}^{i}\right) \eta_{k}^{i}}{\lambda_{F A}+P_{D} \lambda_{B} p\left(z_{k}^{j} \mid x_{k}^{i}\right) \eta_{k}^{i}}\right]
\end{aligned}
$$

where $\eta_{k}^{i}=p_{1}\left(a_{k}^{i}\right) / p_{0}\left(a_{k}^{i}\right)$ denotes the amplitude likelihood ratio.

Note that Eq. (15) can be modelled as a graphic model, by considering each association event as a node. For the considered graphic model, Gibbs sampling is leveraged here to find approximate marginal association probability. This strategy can directly avoid enumerating all possible joint events and thus is computational efficient. Finally, providing the marginal association probability $p\left(\theta_{k} \mid Z_{k}\right)$, local featureaided JPDA can be implemented via Eqs. (8)-(11).

Remark 1. It is clear from Eq. (15) that the amplitude likelihood ratio $\eta_{k}^{i}$ affects the probability terms $P_{D} p\left(\chi_{k}^{i} \mid Z_{k}\right) p\left(\tilde{z}_{k}^{j} \mid x_{k}^{i}\right) \eta_{k}^{i} \quad$ (e.g., existing targets) and $P_{D} \lambda_{B} p\left(\tilde{z}_{k}^{j} \mid x_{k}^{i}\right) \eta_{k}^{i}$ (e.g., new birth targets) by favouring a high amplitude measurement. Therefore, a strong measurement will receive a higher probability of being a target by the tracker. This could enable the improvement of data association and track initialisation in low SNR scenarios, where the detection probability is low.

\section{B. Algorithm Summary}

Algorithm 1 summarises the proposed feature enhanced local JPDA filter discussed above.

Algorithm 1 Feature enhanced local JPDA filter

Input: The previous fused estimation $\left\{x_{k-1 \mid k-1}^{i}, P_{k-1 \mid k-1}^{i}\right\}$, current measurement set $Z_{k}^{l}$ of the $l$ th sensor

Output: Current local estimation $\left\{x_{k \mid k}^{i, l}, P_{k \mid k}^{i, l}\right\}$ of the $l$ th sensor

1: function LoCAL_JPDA $\left(\left\{x_{k-1 \mid k-1}^{i}, P_{k-1 \mid k-1}^{i}\right\}, Z_{k}^{l}\right)$
2: $\quad$ Predict the existed tracks using target dynamics
3: $\quad$ Update each track with all possible measurements by
Kalman filter
4: Calculate the marginal probability $p\left(\theta_{k}^{i} \mid Z_{k}^{l}\right)$ using
Gibbs sampling
5: Calculate the existence-conditioned marginal probabil-
ity $p\left(\theta_{k}^{i} \mid \chi_{k}^{i}, Z_{k}^{l}\right)$
6: Forming a new track for each measurement
7: Calculate the target state estimation
8: Perform track confirmation and deletion based on
existence probability
9: end function

\section{TRACK-TO-TRACK FUSION}

Unlike single target sensor fusion, track-to-track association is required to find the same source origins of all local tracks that are from different sensors for multi-target tracking scenarios. Typical track-to-track association utilises the so-called $S-D$ formulation, which is similar to trackto-measurement multi-dimensional assignment in MHT algorithm. The main difference between track-to-track association and measurement-to-track association is the formulation of association cost function. It is known that when the number 
of sensors is larger than 2, the $S-D$ association is NPhard and thus exact solution is tractable only for some smallscale problems. To this end, we propose a novel efficient track-to-track association strategy using a modified DBSCAN clustering algorithm. For simplicity, the proposed track-totrack association algorithm is designed based on the ideal environmental condition, e.g., no communication delay and packet loss. However, the effect of these physical constraints will be empirically analysed in simulations.

\section{A. Modified DBSCAN Clustering Using Statistical Test}

Intuitively, if the estimated tracks from different sensors are close enough, it is logical to consider that they originate from the same target. From this rational, it should be possible to exploit clustering algorithm in finding different local estimated tracks that have the same origin. The original DBSCAN clustering algorithm [32] is an efficient data clustering algorithm to group together points that are closely packed together using Euclidean distance and to sort out outliers that lie alone in low-density regions. The advantage of DBSCAN clustering is that it requires no knowledge on the number of clusters and, hence, is suitable for the target tracking applications. However, due to unperfect sensors and environmental noises, the local estimations always contain uncertainties and, thereby, the simple DBSCAN might not perform well for target tracking applications. To mitigate the effect of estimation uncertainty on track-to-track fusion, we embed the statistical test into DBSCAN clustering for a more reliable track-to-track fusion scheme.

Without loss of generality, we consider the track-to-track association as a hypothesis testing problem. For the purpose of illustration, we assume that the true target state $x_{k}^{i}$ is related to the local tracks $x_{k}^{i, l_{1}}$ and $x_{k}^{i, l_{2}}$ of the $l_{1}$ th and $l_{2}$ th sensors. Denote $\mathcal{H}_{12}$ as the hypothesis that the local tracks $x_{k}^{i, l_{1}}$ and $x_{k}^{i, l_{2}}$ come from the same target. Then, we have the hypothesis probability as

$$
\Lambda\left(\mathcal{H}_{12}\right)=p\left(x_{k}^{i, l_{1}}, x_{k}^{i, l_{2}} \mid x_{k}^{i}\right)
$$

Without any knowledge of the sensing environment, all tracks in the sensor's field-of-view (FOV) are equally likely. Then, (16) can be reduced to

$$
\begin{aligned}
\Lambda\left(\mathcal{H}_{12}\right) & =p\left(x_{k}^{i, l_{1}}, x_{k}^{i, l_{2}} \mid x_{k}^{i}\right) \\
& =p\left(x_{k}^{i, l_{2}} \mid x_{k}^{i, l_{1}}\right) p\left(x_{k}^{i, l_{1}} \mid x_{k}^{i}\right) \\
& =p\left(x_{k}^{i, l_{2}} \mid x_{k}^{i, l_{1}}\right) p\left(x_{k}^{i, l_{1}}\right) \\
& =\frac{1}{V_{s}} p\left(x_{k}^{i, l_{2}} \mid x_{k}^{i, l_{1}}\right)
\end{aligned}
$$

Note that we utilise the track from the $l_{1}$ th sensor as the reference track in (17). It is obviously legitimate that any other tracks can be used as reference track. Assuming that $p\left(x_{k}^{i, l_{1}} \mid x_{k}^{i}\right)$ and $p\left(x_{k}^{i, l_{2}} \mid x_{k}^{i}\right)$ are Gaussian, we can obtain the analytical solution of $\Lambda\left(\mathcal{H}_{12}\right)$ as

$$
\begin{aligned}
& \Lambda\left(\mathcal{H}_{12}\right)=\frac{1}{V_{s}} p\left(x_{k}^{i, l_{2}} \mid x_{k}^{i, l_{1}}\right) \\
& =\frac{1}{V_{s}} \mathcal{N}\left(x_{k}^{i, l_{2}} ; x_{k}^{i, l_{1}}, P_{k}^{l_{1}}+P_{k}^{l_{2}}-P_{k}^{l_{1}, l_{2}}-\left(P_{k}^{l_{1}, l_{2}}\right)^{T}\right) \\
& =\frac{1}{V_{s}} \mathcal{N}\left(x_{k}^{i, l_{2}}-x_{k}^{i, l_{1}} ; 0, P_{k}^{l_{1}}+P_{k}^{l_{2}}-P_{k}^{l_{1}, l_{2}}-\left(P_{k}^{l_{1}, l_{2}}\right)^{T}\right)
\end{aligned}
$$

where $P_{k}^{l_{1}, l_{2}}$ denotes the cross-covariance between these two estimated tracks, resulting from the common process noise, and can be obtained by a recursive update or the approximated approach shown in [33].

Based on (18), the statistical distance between $x_{k \mid k}^{i, l_{1}}$ and $x_{k \mid k}^{i, l_{2}}$ can be formulated as

$$
\begin{aligned}
& D\left(x_{k}^{i, l_{1}}, x_{k}^{i, l_{2}}\right)=\left(x_{k}^{i, l_{2}}-x_{k}^{i, l_{1}}\right)^{T} \\
& \times\left(P_{k}^{l_{1}}+P_{k}^{l_{2}}-P_{k}^{l_{1}, l_{2}}-\left(P_{k}^{l_{1}, l_{2}}\right)^{T}\right)^{-1}\left(x_{k}^{i, l_{2}}-x_{k}^{i, l_{1}}\right)
\end{aligned}
$$

which is also known as the $\chi^{2}$ based common origin test.

As the statistical distance $D\left(x_{k}^{i, l_{1}}, x_{k}^{i, l_{2}}\right)$ can be utilised as a similarity measure between two local estimations, we embed $D\left(x_{k}^{i, l_{1}}, x_{k}^{i, l_{2}}\right)$ into the original DBSCAN clustering method for track-to-track association. If the statistical distance $D\left(x_{k}^{i, l_{1}}, x_{k}^{i, l_{2}}\right)$ between two local estimated tracks from different sensors satisfies the similarity condition, i.e., $D\left(x_{k}^{i, l_{1}}, x_{k}^{i, l_{2}}\right) \leq \varepsilon$, we confirm that these two tracks have the same origin.

Note that the original DBSCAN clustering used a tune parameter minPts, known as the minimum number of points required to form a dense region, to find the outliers. In our case, as the measurement noise and false alarms are tackled by the local JPDA filter, we assume that track-to-track fusion is performed under outlier-free condition and hence one can simply adopt minPts $=1$ here. Noting that each sensor has its own FOV limit, a target, therefore, might be observed by only one sensor. This means that minPts $=1$ also has practical meaning. Since minPts $=1$, it is clear that the proposed modified DBSCAN clustering has only one tuning parameter, i.e. the similarity threshold $\varepsilon$, and thus the clustering performance is highly dependent on the selection of $\varepsilon$. On one hand, if $\varepsilon$ is too large, the estimated tracks from different targets might be clustered into one group, leading to target miss detection, especially when targets are moving closely. On the other hand, small $\varepsilon$ will enforce the estimated tracks that have the same origin into different clusters. This reveals that the similarity threshold $\varepsilon$ is a trade-off tuning parameter between miss detection and clustering accuracy. To tackle this problem and improve the clustering accuracy, we enforce the following constraint for each cluster.

Constraint. The local estimations obtained by the same sensor cannot be clustered into the same cluster. 
Note that this constraint is helpful in fusing closelyspaced local estimates. Consider a scenario, where two sensors are monitoring two closely moving targets. Assume at time instant $k$ sensor 1 provides local estimates $x_{k}^{1,1}, x_{k}^{2,1}$ and sensor 2 generates local estimates $x_{k}^{1,2}, x_{k}^{2,2}$. Clearly, if conditions $D\left(x_{k}^{1,1}, x_{k}^{1,2}\right) \leq \varepsilon, D\left(x_{k}^{1,1}, x_{k}^{2,2}\right)>\varepsilon$ are satisfied, DBSCAN clustering can identify the two targets. When $D\left(x_{k}^{1,1}, x_{k}^{1,2}\right) \leq \varepsilon, D\left(x_{k}^{1,1}, x_{k}^{2,2}\right) \leq \varepsilon$, the aforementioned hard constraint will enforce the fusion either between $x_{k}^{1,1}$ and $x_{k}^{1,2}$ or between $x_{k}^{1,1}$ and $x_{k}^{2,2}$. In other words, only one local estimate from sensor 2 can be utilised for fusion with $x_{k}^{1,1}$. Therefore, by imposing the preceding constraint, the elements of each cluster are the local estimations with different sensor index and thus each cluster represents a specific target. With these in mind, we summarise the pseudocode of the proposed modified DBSCAN clustering algorithm using statistical test in Algorithm 2.

Remark 2. It is known that the original $S-D$ assignment problem amounts to a linear binary programming problem with $\mathcal{O}\left(N_{k}^{N_{s}}\right)$ variables and $\mathcal{O}\left(N_{k} N_{s}\right)$ constrains. Therefore, the complexity of the problem increases exponentially with the number sensors. The mainstream Lagrangian relaxation algorithm iteratively solves a $2-D$ assignment problem with complexity $\mathcal{O}\left(\left[N_{s} N_{k}\right]^{3}\right)$ [26], [27]. This means that the complexity of this algorithm is given by $\mathcal{O}\left(N_{i t e}\left[N_{s} N_{k}\right]^{3}\right)$, where $N_{i t e}$ denotes the number of iterations. As a comparison, since the neighbourhood query in DBSCAN clustering executes in $\mathcal{O}\left(\log \left[N_{s} N_{k}\right]\right)$ [32], the overall average runtime complexity is given by $\mathcal{O}\left(N_{s} N_{k} \log \left[N_{s} N_{k}\right]\right)$. This reveals that the proposed track-to-track algorithm is less computationally complex compared with the exact $S-D$ algorithm and the well-known Lagrangian relaxation approach.

\section{B. Local Estimation Fusion}

Once we obtain the clustering results, the next step is to fuse the local estimated tracks to generate the global estimation for each cluster. For multi-sensor system, the fused estimation is a mixture distribution. Generally, propagation of the mixture distribution is computationally intractable due to the explosion of mixture terms. An alternative solution is to find a single candidate distribution to approximate the mixture distribution. Therefore, the estimation fusion itself is a mixture reduction problem.

Here, the GCI approach is utilised to fuse the estimations. The advantage of GCI lies in its robustness against the unknown correlations among the information sources. For local estimations $\left\{x_{k \mid k}^{i, l}, P_{k \mid k}^{i, l}\right\}$, the closed-form solution of GCI is given by [34]

$$
\begin{aligned}
& P_{k \mid k}^{i}=\left[\sum_{l=1}^{N_{s}} \omega_{l}^{i}\left(P_{k \mid k}^{i, l}\right)^{-1}\right]^{-1} \\
& x_{k \mid k}^{i}=P_{k \mid k}^{i} \sum_{l=1}^{N_{s}} \omega_{l}^{i}\left(P_{k \mid k}^{i, l}\right)^{-1} x_{k \mid k}^{i, l}
\end{aligned}
$$

where $\omega_{l}^{i}$ denotes the weightings.

It should be noted that the the track continuity or the target identity is lost during the clustering process, i.e., target ID cannot be maintained during clustering. In order to maintain track continuity, we apply the simple bi-partition matching algorithm [35], [36] to every consecutive two scans. This choice is motivated by the fact that we do not need to handle the clutter issue and sensor noise problem at this stage as they have already been treated by the local JPDA filter.

\section{Algorithm Summary}

Algorithm 3 summarises the proposed multi-sensor multitarget tracking with feature augmentation and clustering discussed above.

\section{Numerical Simulations}

In this section, the effectiveness of the proposed multisensor multi-target tracking algorithm is demonstrated through numerical simulations in a cluttered environment. Our experiments explore a scenario, shown in Fig. 3, that consists of 4 sensors and 10 moving targets with different birth times. To accommodate the nonlinear measurements, the well-known UKF is utilised in the local JPDA filter.

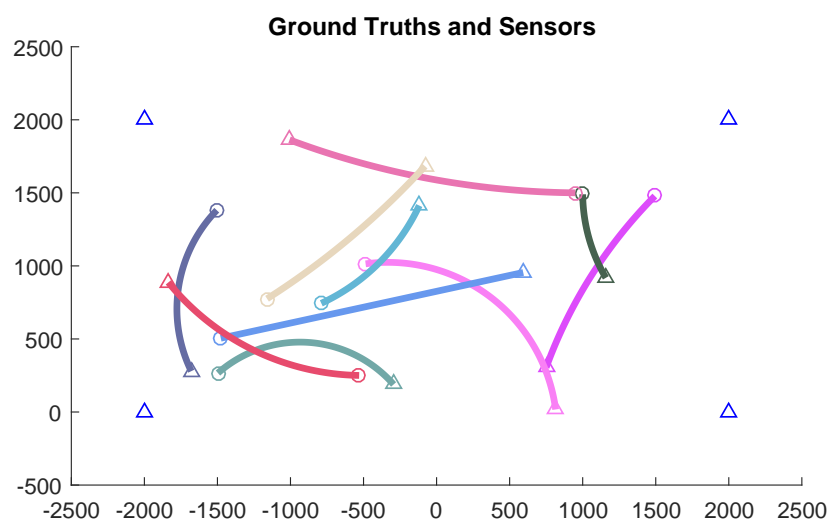

Fig. 3: Ground truth and sensor locations.

\section{A. Simulation Setup}

The state vector contains planar position and velocity. We use the well-known coordinated turning (CT) model for target prediction. The CT model is defined as

$$
x_{k}=F x_{k-1}+G w_{k-1}
$$

with

$F \triangleq\left[\begin{array}{cccc}1 & \frac{\sin \left(\omega_{k} T\right)}{\omega_{k}} & 0 & -\frac{1-\cos \left(\omega_{k} T\right)}{\omega_{k}} \\ 0 & \cos \left(\omega_{k} T\right) & 0 & -\sin \left(\omega_{k} T\right) \\ 0 & \frac{1-\cos \left(\omega_{k} T\right)}{\omega_{k}} & 1 & \frac{\sin \left(\omega_{k} T\right)}{\omega_{k}} \\ 0 & \sin \left(\omega_{k} T\right) & 0 & \cos \left(\omega_{k} T\right)\end{array}\right], G \triangleq\left[\begin{array}{c}T^{2} / 2 \\ T \\ T^{2} / 2 \\ T\end{array}\right]$

where $T=1 s$ denotes the sampling period and $w_{k} \sim$ $\mathcal{N}\left(\cdot ; 0, \sigma_{v}^{2}\right)$ is the Gaussian process noise with $\sigma_{v}=15 \mathrm{~m} / \mathrm{s}^{2}$. $\omega_{k}$ stands for the turning rate, which is assumed to be a random walk process as $\omega_{k+1}=\omega_{k}+\epsilon_{k}$ with $\epsilon_{k} \sim \mathcal{N}\left(\cdot ; 0, \sigma_{\omega}^{2}\right)$. 

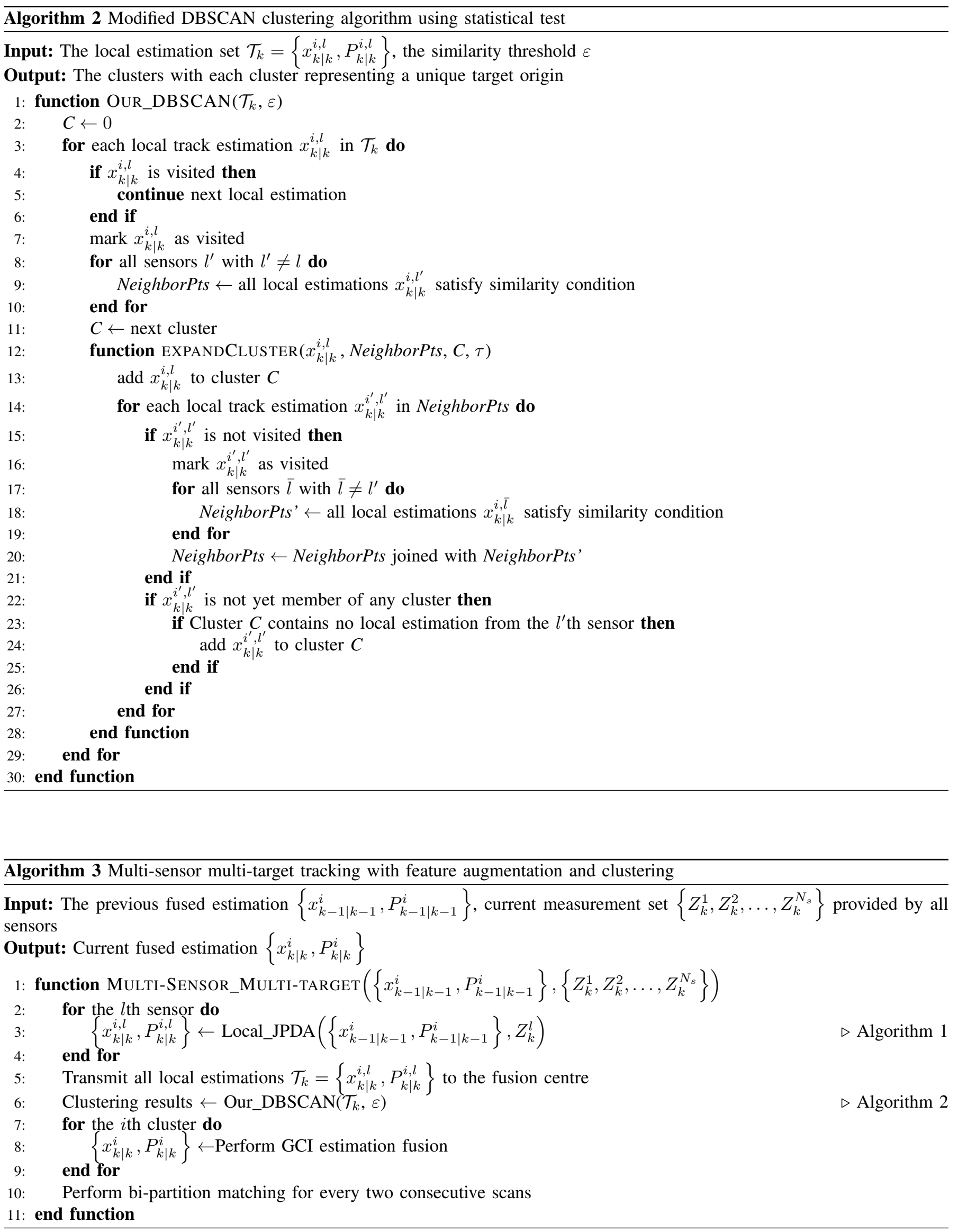
The standard deviation of the random walk process is chosen as $\sigma_{\omega}=3^{\circ}$.

The target-generated nonlinear range and bearing measurements are modelled by

$$
\tilde{z}_{k}=\left[\begin{array}{c}
r_{k} \\
\alpha_{k}
\end{array}\right]=\left[\begin{array}{c}
\sqrt{\left(x_{T, k}-x_{s}\right)^{2}+\left(y_{T, k}-y_{s}\right)^{2}} \\
\arctan \left(\frac{y_{T, k}-y_{s}}{x_{T, k}-x_{s}}\right)
\end{array}\right]+v_{k}
$$

where $\left(x_{T, k}, y_{T, k}\right)$ is target position, $\left(x_{s}, y_{s}\right)$ sensor position, and $v_{k} \sim \mathcal{N}\left(\cdot ; 0, R_{k}\right)$ the Gaussian measurement noise with $R_{k}=\operatorname{diag}\left(\sigma_{r}^{2}, \sigma_{a}^{2}\right)$. The standard deviations of the measurement noise are selected as $\sigma_{r}=5 m, \sigma_{a}=1^{\circ}$.

The clutter is assumed to be uniformly distributed in the surveillance region with its number being Poisson with $N_{F A}$ average returns at each scan. Gating is performed with a threshold such that the gating probability is $P_{G}=0.999$. A tentative track is confirmed if the existence probability satisfies $p\left(\chi_{k}^{i} \mid Z_{k}\right) \geq 0.8$ and a confirmed track is deleted immediately once $p\left(\chi_{k}^{i} \mid Z_{k}\right) \leq 0.1$. For simplicity, we assume that all sensors have equal properties and thus have the same fusion weighting coefficient, i.e. $\omega_{l}^{i}=1 / N_{s}$, in our cases. For running the proposed modified DBSCAN algorithm, the similarity threshold $\varepsilon$ is selected as $\varepsilon=5$. In order to show the advantage of using amplitude feature augmentation, we consider a low SNR environment with $\mathrm{SNR}=10 \mathrm{~dB}$. Note that the target detection is obtained by thresholding the signal with $\tau$. However, there is no theoretical way to tune this parameter. In simulations, we utilise the empirical convention [18] that

$$
\tau=-\log \left[12 \sigma_{m}^{2} \lambda_{F A}\right]
$$

where $\sigma_{m}$ denotes the standard deviation of position measurements.

In order to apply (24) to the nonlinear angle and range measurement case, it is required to convert the standard deviations of the polar measurements $\sigma_{r}, \sigma_{a}$ to the Cartesian one $\sigma_{m}$. One typical way of solving the measurement converting problem is the well-known extended Kalman filter. However, this technique utilises a simple first-order approximation, which includes the potential threat of inconsistency between the calculated error covariance and the true error. To avoid this pitfall, we leverage the recently proposed unbiased converted measurement (UCM) [37] in our study. The converted position measurement covariance matrix of $\mathrm{UCM}$ is given by

$$
\begin{aligned}
R_{k}^{11}= & \frac{1}{2}\left(r_{k}^{2}+\sigma_{r}^{2}\right)\left[1+\cos \left(2 \alpha_{k}\right) e^{-2 \sigma_{a}^{2}}\right]-e^{\sigma_{a}^{2}} r_{k}^{2} \cos ^{2}\left(\alpha_{k}\right) \\
R_{k}^{22}= & \frac{1}{2}\left(r_{k}^{2}+\sigma_{r}^{2}\right)\left[1-\cos \left(2 \alpha_{k}\right) e^{-2 \sigma_{a}^{2}}\right]-e^{\sigma_{a}^{2}} r_{k}^{2} \sin ^{2}\left(\alpha_{k}\right) \\
R_{k}^{12}= & R_{k}^{21}=\frac{1}{2}\left(r_{k}^{2}+\sigma_{r}^{2}\right)\left[\sin \left(2 \alpha_{k}\right) e^{-2 \sigma_{a}^{2}}\right] \\
& -e^{\sigma_{a}^{2}} r_{k}^{2} \cos \left(\alpha_{k}\right) \sin \left(\alpha_{k}\right)
\end{aligned}
$$

For simplicity, we ignore the correlated term $R_{k}^{12}$ and choose the standard deviation of the position measurements $\sigma_{m}$ as $\sigma_{m}^{2}=\max \left\{R_{k}^{11}, R_{k}^{22}\right\}$, which represents the maximum uncertainty in the converted position measurements.

The optimal sub-pattern assignment (OSPA) distance metric [38] is considered here for overall evaluation of performance, namely, cardinality and position estimation errors. Let $X$ and $Y$ be the position estimation set and true target position set, respectively. The cardinality of these two sets are $m$ and $n$, respectively. Then, for $c>0$ and $1 \leq p<\infty$, the OSPA distance $d_{p}^{c}(X, Y)$ is defined as [38]

$$
\begin{aligned}
& d_{p}^{c}(X, Y) \triangleq \\
& \begin{cases}{\left[\frac{1}{n}\left(\min _{\pi \in \Pi_{n}} \sum_{i=1}^{m} d^{c}\left(x_{i}, y_{\pi(i)}\right)^{p}+c^{p}(n-m)\right)\right]^{1 / p},} & m \leq n \\
d_{p}^{c}(Y, X), & m>n\end{cases}
\end{aligned}
$$

where $\Pi_{n}$ denotes the set of all permutations on $\{1,2, \ldots, n\}$ for any positive integer $n . d^{c}\left(x_{i}, y_{\pi(i)}\right)=$ $\min \left(d\left(x_{i}, y_{\pi(i)}\right), c\right)$ is the cut-off Euclidean distance between two vectors with $d\left(x_{i}, y_{\pi(i)}\right)$ being the Euclidean distance. The order parameter $p$ determines the sensitivity of $d_{p}^{c}(X, Y)$ in penalizing estimation outliers, while the cut-off parameter $c$ determines the relative weighting of the penalties allocated to cardinality and localization errors. In all simulations, these two parameters are set as $p=1, c=100$.

\section{B. Simulation Results}

To show the effectiveness of the proposed algorithm, the following different testing cases are considered in simulations.

1) With/without amplitude feature: To show the advantages of utilising amplitude feature, Figs. 4 (a) and 4 (b) compare the mean OSPA distances of 100 Monte-Carlo runs between the fused estimation and local estimations. Figure 4 (a) shows the results that never consider the amplitude information while Fig. 4 (b) is for amplitude feature enhanced estimation. These two figures clearly reveal that the fused estimation greatly improves the tracking performance (approximately 60\% improvement) in terms of OSPA accuracy whether or not we consider the amplitude augmentation. In order to demonstrate that the effect of the feature information on the tracking performance, Fig. 5 depicts the comparisons of the mean OSPA distances between trackers with and without feature augmentation. From this figure, one can observe that utilising the detection amplitude provides more accurate estimations. Notably, augmenting the original JPDA with the amplitude knowledge significantly increases the track confirmation speed for a low SNR environment.

2) Variation of SNR: In MTT, the clutter rate plays an important role in governing the overall estimation performance. Figure 6 shows the mean OSPA distance of the proposed algorithm with respect to different SNR $=7 d B, 10 d B, 13 d B$. Intuitively, higher SNR environment will generate more clutters with the same detection threshold $\tau$, increasing the possibility of data association failure. Therefore, the performance will degrade with lower SNR. This can be clearly observed from Fig. 6. However, by utilising the detection amplitude as the domain knowledge, the performance of the proposed algorithm does not show much difference even in a low SNR environment, demonstrating the strong robustness against the variation of SNR or clutter rate. 


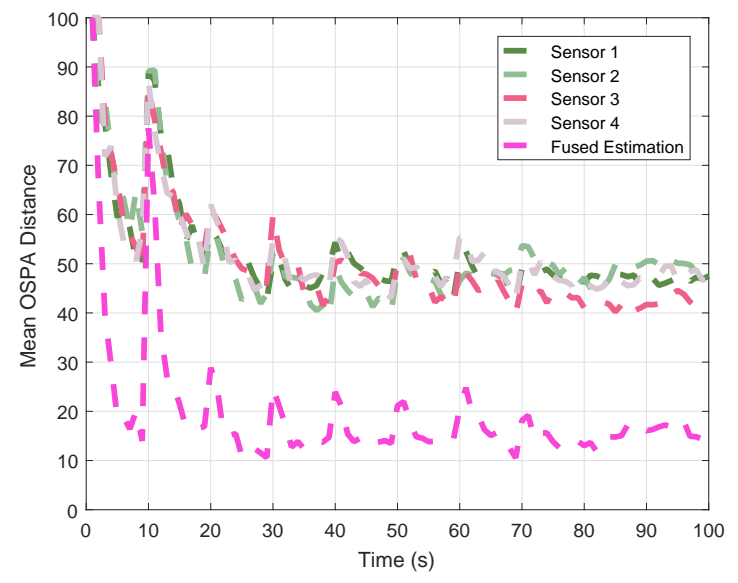

(a) Without amplitude feature

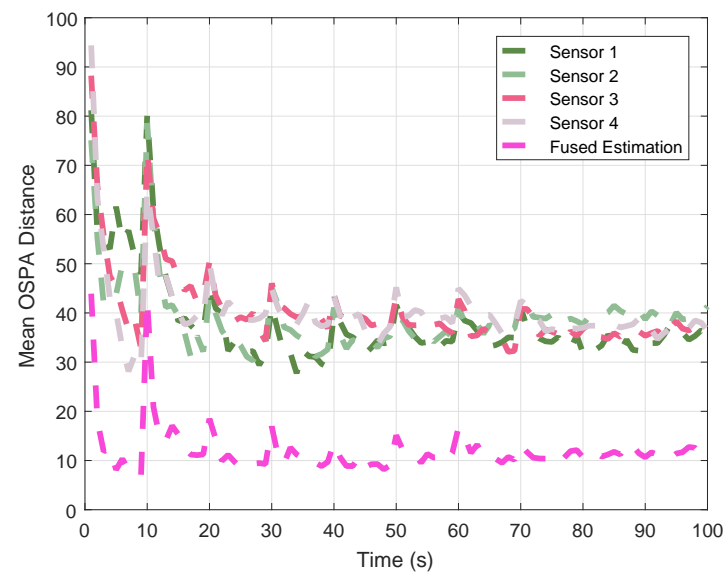

(b) With amplitude feature

Fig. 4: Mean OSPA distance.

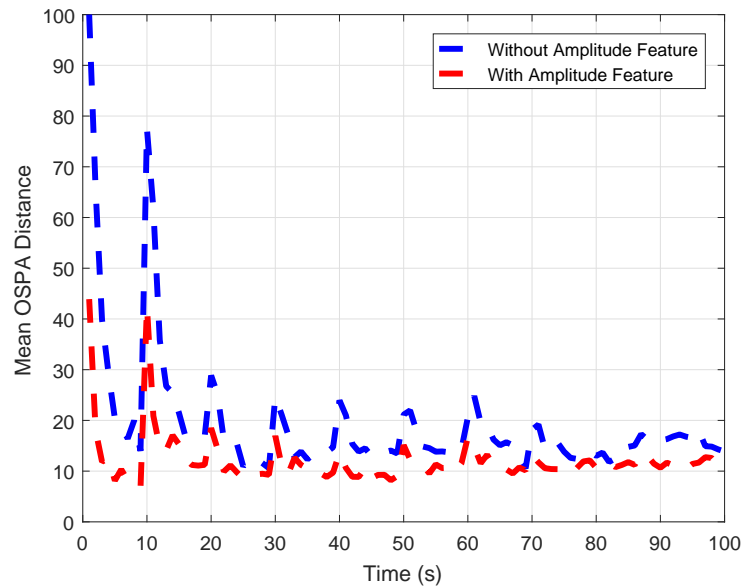

Fig. 5: Mean OSPA distance comparison.

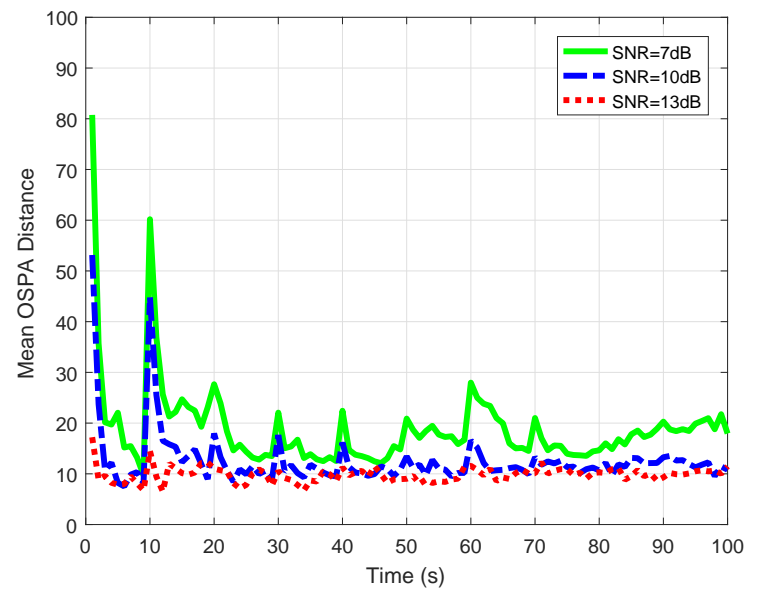

Fig. 6: Mean OSPA distance with respect to different SNRs.
3) Computational complexity: To further show the advantages of the proposed approach in terms computation complexity, comparisons are made with exact $S-D$ solution and approximate Lagrange relaxation algorithms. The mean OSPA distances of 100 Monte-Carlo runs obtained from three different algorithms are presented in Fig. 7. The quantitative comparison results of average OSPA distance over time and running time are provided in Table I. From Fig. 7, one can note that the exact $S-D$ solution provides the best tracking performance among these three different algorithms. However, the exact $S-D$ solution is highly computational expensive, as confirmed by the running time shown in Table I. Even though the approximate Lagrange relaxation executes within an acceptable time, it provides the worst tracking accuracy in terms of OSPA distance. Compared to exact $S-D$ solution and Lagrange relaxation approach, the proposed algorithm achieves reasonable accuracy with lower computational demand.

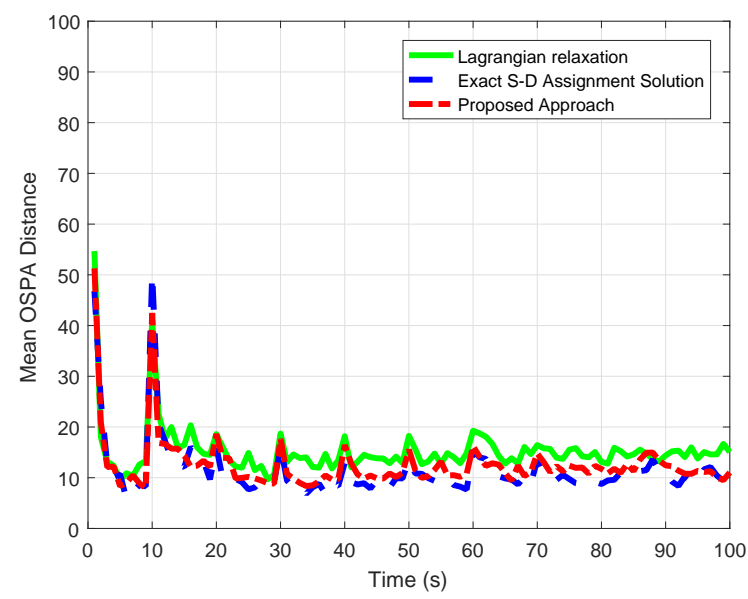

Fig. 7: Mean OSPA distance comparison.

4) Communication delay: Practically, communications between the fusion centre and sensor nodes usually suffer from time delays. We investigate the effect of time delay on the 
TABLE I: Average OSPA distance and running time comparisons.

\begin{tabular}{c|ccc}
\hline \hline & Exact $S-D$ & Lagrange relaxation & Proposed approach \\
\hline Mean OSPA & 11.2810 & 15.2237 & 12.4820 \\
Running time & $469.31 \mathrm{~s}$ & $36.19 \mathrm{~s}$ & $21.51 \mathrm{~s}$ \\
\hline
\end{tabular}

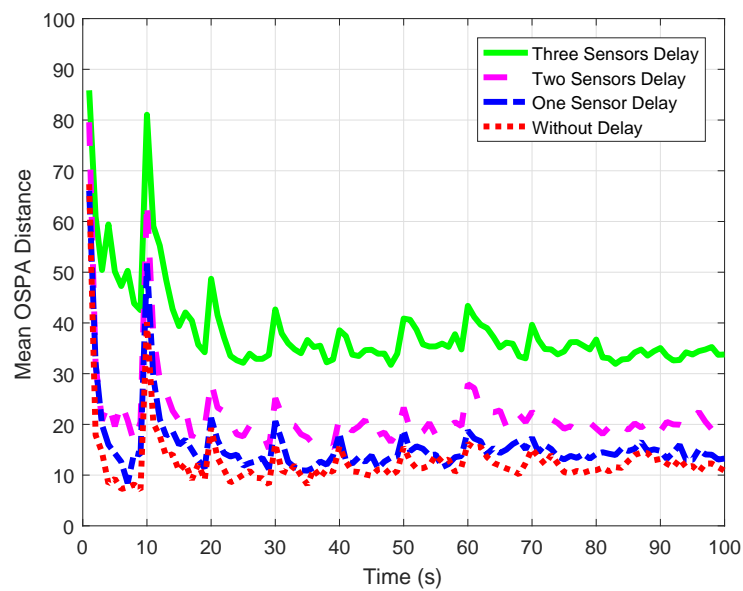

Fig. 8: Mean OSPA distance with communication delay.

performance of proposed algorithm. To this end, four different cases are considered here: (1) without delay; (2) one sensor delay; (3) two sensors delay; and (4) three sensors delay. Here, cases (2)-(4) consider one-three sensors that cannot communicate with the fusion centre within the sampling period due to communication delay. Figure 8 presents the performance of the proposed algorithm with respect to different cases. From this figure, it is obvious that the performance of the proposed algorithm improves with less communication delay. This can be attributed to the fact that more information is gained from sensor nodes with less communication loss, thus leading to performance improvement in information fusion. However, unless there is certain amount of information for fusing, e.g., cases (2)-(3), the performance of the proposed algorithm does not differ much from the ideal case, e.g., case (1). This demonstrates that the proposed algorithm can tolerant certain degree of communication loss and time delay.

5) Limited sensor FOV: In reality, every sensor has its own FOV and thus a target cannot be detected by a sensor if the target is out of the sensor's FOV. To show the effectiveness of the proposed algorithm with limited sensor coverage, simulations are performed for the case: 6 sensors with detection range $1800 \mathrm{~m}$ and limiting sensing angles $90^{\circ}$. The ground truth and sensor locations of this case is shown in Fig. 9, where colour solid lines are target true trajectories, the blue dashed lines the sensor' FOV and the blue triangles the sensor locations. Figure 10 compares the mean OSPA distances of 100 Monte-Carlo runs between the fused estimation and local estimations. From this figure, it is clear that single sensor with limited FOV cannot provide reliable tracking results due to target loss. As a comparison, the fused estimation still provide reasonably good tracking performance through information communication among all sensors.

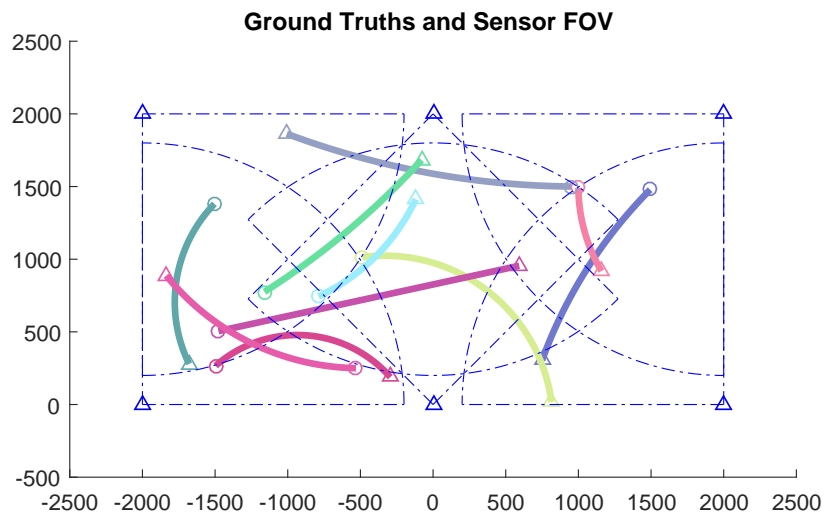

Fig. 9: Ground truth and sensor locations.

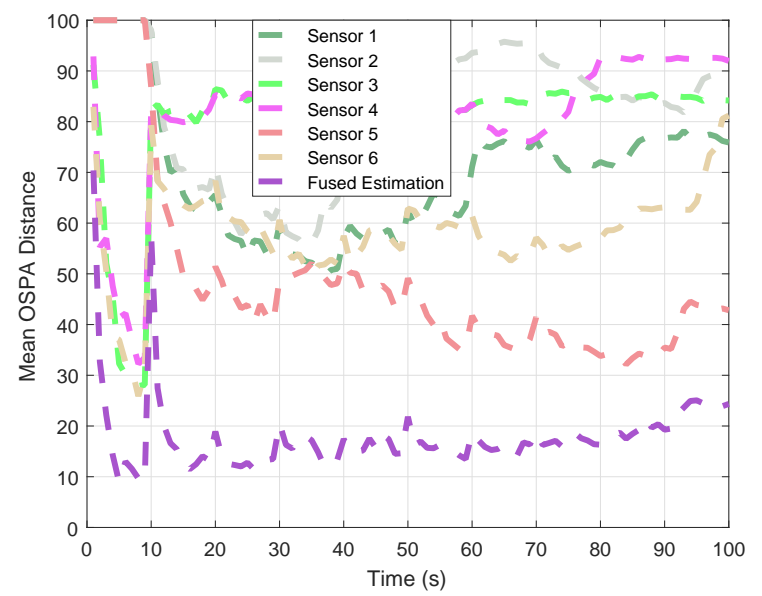

Fig. 10: Mean OSPA distance with limited sensor FOV.

\section{CONCLUSIONS}

We have proposed a multi-sensor multi-target tracking algorithm using an feature enhanced local JPDA filter in conjunction with a DBSCAN clustering-based track-to-track fusion scheme. Unlike classical JPDA filter, the proposed local filter utilises the detection amplitude augmentation as the domain knowledge in measurement-to-track association. As confirmed by the simulation results, utilising the amplitude knowledge is beneficial for track confirmation and maintenance in a low SNR environment. In the fusion stage, we embed the statistical test into the DBSCAN clustering algorithm to group the local tracks that originate from the same target and the GCI approach is leveraged to fuse the local estimations of each cluster. Simulation results clearly show that the proposed algorithm is a competitive alternative for multi-sensor multitarget tracking.

\section{REFERENCES}

[1] X. Chen, L. An, and B. Bhanu, "Multitarget Tracking in Nonoverlapping Cameras Using a Reference Set," IEEE Sensors Journal, vol. 15, no. 5, pp. 2692-2704, 2015.

[2] A. Dallil, M. Oussalah, and A. Ouldali, "Sensor Fusion and Target Tracking Using Evidential Data Association," IEEE Sensors Journal, vol. 13, no. 1, pp. 285-293, 2013.

[3] P. Zong and M. Barbary, "Improved Multi-Bernoulli Filter for Extended Stealth Targets Tracking Based on Sub-Random Matrices," IEEE Sensors Journal, vol. 16, no. 5, pp. 1428-1447, 2016. 
[4] B. Gulmezoglu, M. B. Guldogan, and S. Gezici, "Multiperson tracking with a network of ultrawideband radar sensors based on gaussian mixture PHD filters," IEEE Sensors Journal, vol. 15, no. 4, pp. 2227-2237, 2015.

[5] D. Reid, "An algorithm for tracking multiple targets," IEEE Transactions on Automatic Control, vol. 24, no. 6, pp. 843-854, 1979.

[6] S. Blackman, "Multiple hypothesis tracking for multiple target tracking," IEEE Aerospace and Electronic Systems Magazine, vol. 19, no. 1, pp. $5-18,2004$

[7] S. He, H.-S. Shin, and A. Tsourdos, "Track-oriented multiple hypothesis tracking based on tabu search and gibbs sampling," IEEE Sensors Journal, vol. 18, no. 1, pp. 328-339, 2017.

[8] I. J. Cox and S. L. Hingorani, "Efficient implementation of Reid's multiple hypothesis tracking algorithm and its evaluation for the purpose of visual tracking," IEEE Transactions on Pattern Analysis and Machine Intelligence, vol. 18, no. 2, pp. 138-150, 1996.

[9] J. Areta, Y. Bar-Shalom, M. Levedahl, and K. R. Pattipati, "Hierarchical track association and fusion for a networked surveillance system," Journal of Advances in Information Fusion, vol. 1, no. 2, 2006.

[10] C. Kim, F. Li, A. Ciptadi, and J. M. Rehg, "Multiple hypothesis tracking revisited," Proceedings of the IEEE International Conference on Computer Vision, vol. 11-18-Dece, pp. 4696-4704, 2016.

[11] T. E. Fortmann, Y. Bar-Shalom, and M. Scheffe, "Sonar tracking of multiple targets using joint probabilistic data association," IEEE Engineering of Oceanic Engineering, vol. 8, no. 3, pp. 173-184, 1983.

[12] S. H. Rezatofighi, A. Milan, Z. Zhang, Q. Shi, A. Dick, and I. Reid, "Joint probabilistic data association revisited," Proceedings of the IEEE International Conference on Computer Vision, vol. 11-18-Dece, pp. 3047-3055, 2016.

[13] M. J. Gualdoni, J. S. McCabe, and K. J. DeMars, "An Investigation of Probabilistic Data Association Filters for Multiple Space Object Tracking," AIAA/AAS Astrodynamics Specialist Conference, no. September, pp. 1-20, 2016.

[14] J. Stauch, M. Jah, J. Baldwin, T. Kelecy, and K. A. Hill, "Mutual Application of Joint Probabilistic Data Association, Filtering, and Smoothing Techniques for Robust Multiple Space Object Tracking," AIAA/AAS Astrodynamics Specialist Conference, no. August, pp. 1-21, 2014.

[15] N. N. Kachouie and P. W. Fieguth, "Extended-Hungarian-JPDA: Exact single-frame stem cell tracking," IEEE Transactions on Biomedical Engineering, vol. 54, no. 11, pp. 2011-2019, 2007.

[16] Y. Bar-Shalom, T. Kirubarajan, and X. Lin, "Probabilistic data association techniques for target tracking with applications to sonar, radar and EO sensors," IEEE Aerospace and Electronic Systems Magazine, vol. 20, no. 8 II, pp. 37-54, 2005.

[17] D. Lerro, N. Underwater, and N. London, "Tracking with Target Amplitude Feature," IEEE Transactions on Aerospace and Electronic Systems, vol. 29, no. 2, 1993.

[18] P. Willett, R. Niu, and Y. Bar-Shalom, "Integration of Bayes detection with target tracking," IEEE Transactions on Signal Processing, vol. 49, no. 1, pp. 17-29, 2001.

[19] L. Hong, N. Cui, M. Pronobis, and S. Scott, "Local motion feature aided ground moving target tracking with GMTI and HRR measurements," IEEE Transactions on Automatic Control, vol. 50, no. 1, pp. 127-133, 2005.

[20] D. Mušicki, X. Chen, T. L. Song, T. Kirubarajan, and H. H. Lee, "Trackto-track fusion with target existence," IET Radar, Sonar \& Navigation, vol. 9, no. 3, pp. 241-248, 2015.

[21] R. W. Osbome, Y. Bar-Shalom, and P. Willett, "Track-to-track association with augmented state," in 14th International Conference on Information Fusion, July 2011, pp. 1-8.

[22] H. Chen and Y. Bar-Shalom, "Track association and fusion with heterogeneous local trackers," Proceedings of the IEEE Conference on Decision and Control, pp. 2675-2680, 2007.

[23] F. Fkie, F. Strasse, and G. Email, "On Decorrelated Track-to-Track Fusion based on Accumulated State Densities," Information Fusion (FUSION), 2014, pp. 1-6, 2014.

[24] Y. Bar-Shalom and H. Chen, "Multisensor track-to-track association for tracks with dependent errors," 43rd IEEE Conference on Decision and Control, vol. 3, no. 1, pp. 3-14, 2004.

[25] J. Areta, Y. Bar-shalom, and M. Levedahl, "Comparison of Two MDA Algorithms for a Problem in Missile Defense," Proceedings of SPIE, vol. 6236, no. 1, pp. 1-12, 2006.

[26] S. Deb, K. R. Pattipati, and Y. Bar-Shalom, "A multisensor-multitarget data association algorithm for heterogeneous sensors," IEEE Transactions on Aerospace and Electronic Systems, vol. 29, no. 2, pp. 560-568, 1993.
[27] S. Deb, M. Yeddanapudi, K. Pattipati, and Y. Bar-Shalom, "A generalized sd assignment algorithm for multisensor-multitarget state estimation," IEEE Transactions on Aerospace and Electronic Systems, vol. 33 no. 2, pp. 523-538, 1997.

[28] A. B. Poore and A. J. Robertson III, "A New Lagrangian Relaxation Based Algorithm for a Class of Multidimensional Assignment Problems," Computational Optimization and Applications, vol. 8, no. 2, pp. 129-150, 1997

[29] M. I. Skolnik, Introduction to Radar System, 3rd ed. New York: McGraw-Hill, 2002.

[30] D. Lerro and Y. Bar-Shalom, "Automated tracking with target amplitude information," in Proceeding of American Control Conference, San Diego, 1990, pp. 2875-2880.

[31] S. Challa, Fundamentals of object tracking. Cambridge, UK: Cambridge University Press, 2011.

[32] M. Ester, H.-P. Kriegel, J. Sander, and X. Xu, "A density-based algorithm for discovering clusters in large spatial databases with noise," in $K D D$, vol. 96, 1996, pp. 226-231.

[33] L. M. Kaplan, Y. Bar-Shalom, and W. D. Blair, "Assignment costs for multiple sensor track-to-track association," IEEE Transactions on Aerospace and Electronic Systems, vol. 44, no. 2, pp. 655-677, 2008.

[34] G. Battistelli and L. Chisci, "Kullback-leibler average, consensus on probability densities, and distributed state estimation with guaranteed stability," Automatica, vol. 50, no. 3, pp. 707-718, 2014.

[35] K. Shafique and M. Shah, "A noniterative greedy algorithm for multiframe point correspondence," IEEE Transactions on Pattern Analysis and Machine Intelligence, vol. 27, no. 1, pp. 51-65, 2005.

[36] J. Hopcroft and R. Karp, "An $n^{5 / 2}$ Algorithm for Maximum Matchings in Bipartite Graphs," vol. 2, no. 4, pp. 225-231, 1973.

[37] S. Bordonaro, P. Willett, and Y. Bar-Shalom, "Decorrelated unbiased converted measurement Kalman filter," IEEE Transactions on Aerospace and Electronic Systems, vol. 50, no. 2, pp. 1431-1444, 2014.

[38] D. Schuhmacher, B. T. Vo, and B. N. Vo, "A consistent metric for performance evaluation of multi-object filters," IEEE Transactions on Signal Processing, vol. 56, no. 8 I, pp. 3447-3457, 2008.

Shaoming He was born in 1991. He received his MSc degree in Aerospace Engineering from Beijing Institute of Technology in 2016. He is currently a PhD student in the School of Aerospace, Transport and Manufacturing, Cranfield University. His research interests include multi-target tracking, guidance and control.

Hyo-Sang Shin received his BSc from Pusan National University in 2004 and gained an MSc on flight dynamics, guidance and control in Aerospace Engineering from KAIST and a $\mathrm{PhD}$ on cooperative missile guidance from Cranfield University in 2006 and 2010, respectively. He is currently Reader on Guidance, Control and Navigation Systems in Autonomous and Intelligent Systems Group at Cranfield University. His current research interests include multiple target tracking, probabilistic target detection and distributed control of multiple agent systems.

Antonios Tsourdos obtained a MEng in electronic, control and systems engineering from the University of Sheffield (1995), an MSc in systems engineering from Cardiff University (1996), and a PhD in nonlinear robust missile autopilot design and analysis from Cranfield University (1999). He is a Professor of Control Engineering with Cranfield University, and was appointed Head of the Centre for Cyber-Physical Systems in 2013. He was a member of the Team Stellar, the winning team for the UK MoD Grand Challenge (2008) and the IET Innovation Award (Category Team, 2009). 
2018-08-03

\section{Multi-sensor multi-target tracking using domain knowledge and clustering}

He, Shaoming

IEEE

Shaoming He, Hyo-Sang Shin and Antonios Tsourdos. Multi-sensor multi-target tracking using domain knowledge and clustering. IEEE Sensors Journal, Available online 3 August 2018 https://doi.org/10.1109/JSEN.2018.2863105

Downloaded from Cranfield Library Services E-Repository 